Annals of the Japan Association for Philosophy of Science, March 1991

\title{
Connaissance Sensible et Approximation
}

\author{
Jules Vuiluemin
}

Toute connaissance sensible peut être dite approchée en un sens vague. Ce sens ne devient défini qu'en physique où la connaissance sensible porte sur des grandeurs. Je m'efforcerai donc de préciser d'abord les conditions que suppose pour la connaissance physique l'approximation essentielle de son objet. J'examinerai en second lieu les contraintes que l'approximation fait peser sur les observations, les lois et les théories. J'analyserai enfin les conséqences de ces contraintes sur la vérité et sur la signification des énoncés physiques.

\section{Physique et approximation des grandeurs}

Notre système perceptif est comparable à une théorie physique implicite. Au départ nos sens fournissent une information. Appelons-la A. Le système nerveux traite cette information et la met en rapport avec les informations semblables, $\mathbf{A}^{\prime}$, qu'il a recueillies dans le passé. Ces dernières informations $A^{\prime}$ ayant été généralement suivies d'information $B^{\prime}$, le système nerveux alerte l'occurrence de $B$. Trois circonstances fondent l'analogie avec la physique. L'information, attendue aussi bien que reçue, est relative à des seuils en vertu des lois de la psychophysique. Au delà, en deçà de ces seuils, l'organisme reste insensible. En second lieu, l'anticipation du système nerveux évoque la prédiction que permet la loi ou la théorie physiques. Enfin, l'anticipation biologique est suivie généralement d'un renforcement de l'habitude; disons "généralement" en vertu des nécessités de la sélection naturelle. Il arrive cependant que, dans le cas des illusions perceptives, l'organisme, trompé, réagisse à contre-temps. On sait comment, un tel échec éteint un réflexe conditionnel. Renforcement et extinction annoncent au niveau du corps ce que seront la confirmation et l'infirmation au niveau de la science.

L'analogie boîte cependant dès qu'on précise les trois circonstances mentionnées.

La sensation, dit-on d'abord, est une connaissance approchée. Elle l'est en vertu des seuils. Mais prenons garde que, lorsque nous affirmons que la sensation croît comme le logarithme de l'excitation, nous comparons, comme l'ont souvent remarqué justement les critiques de la psychophysique pour en tirer d'ailleurs souvent des conclusions erronées, que nous comparons deux séries de termes hétérogènes. La série des excitants est faite de grandeurs physiques, par exemple de

Collège de France, 11, Place Marcelin-Berthelot, 75231, PARIS CEDEX 05, FRANCE 
poids, kilogrammes, hectogrammes, décigrammes, grammes. Ces grandeurs sont dites extensives ${ }^{1}$ : la mesure de deux poids ajoutés sur le plateau d'une balance est la somme de ces deux poids. La série des sensations est faite de grandeurs intensives. La sensation de poids qu'on éprouve quand on soulève deux kilogrammes est plus intense que celle qu'on éprouve quand on soulève un kilogramme, mais dire qu'elle est double de la première serait dépourvu de sens. La loi de Weber-Fechner établit seulement une fonction qui fait correspondre à toutes les discriminations d'ordre constatées sur la série des sensations une discrimination d'ordre et de quantité sur la série des excitations; la correspondance réciproque n'ayant pas lieu.

Bergson $^{2}$ a soutenu, dans une thèse célèbre, que la perception de l'intensité se réduit, à l'analyse, à la perception d'une qualité différente. Quelque jugement qu'on se fasse sur cette thèse, elle ne ruine pas, à elle seule, la psychophysique. Il se pourrait, en effet, que, lorque nous déclarons que, données trois sensations successives et clairement discernées de poids A, B, C, A est plus intense que B qui est, à son tour, plus intense que $C$, nous ne puissions pas séparer de la relation d'intensité un contexte qualitatif dont nous faisons précisément abstraction lorsque nous parlons de la relation mathématique d'ordre. Il se pourrait, par exemple, que des impressions d'irritation ou même de douleur vinssent mentalement contaminer notre conscience d'intensité croîssante.

Une telle concession n'a rien de gênant, s'il est vrai que toutes les informations que nous recevons par les sens sont qualitatives. D'évidence nul ne conteste que nous percevions des formes. L'animal le plus rudimentaire ne survivrait pas s'il n'organisait et ne localisait pas le contenu de ses sensations dans un espace et un temps sensibles. Mais c'est encore comme des qualités qu'il perçoit places et moments. Le psychophysicien, qui est un physicien, remplace ces qualités sensibles par des grandeurs. S'il conserve une série qualitative, c'est en fixant son attention sur son aspect sériel, c'est-à-dire sur l'ensemble ordonné des classes de discriminations qui la composent, ensemble qui donne lieu à une correspondance bien définie avec une série de grandeurs.

Galilée a proclamé avec éclat ce primat de la grandeur en physique. Mais ceux qui ont pratiqué avant lui l'astronomie de position, que nous rangeons dans la physique, ont accepté le même primat. Ainsi Ptolémée proclame qu'il ne s'occupe pas de qualités matérielles et toujours changeantes comme le blanc, le chaud, le sucré, le doux, mais "de la qualité qui regarde formes et mouvement locaux, en cherchant figure, nombre et grandeur, et aussi lieu et temps"3, toutes choses qui relèvent des mathématiques.

A toute grandeur et à toute composition de grandeurs correspondent respectivement, une fois fixé le choix conventionnel de l'unité, un nombre et une opération numérique. Pas de physique donc, sans mathématique. Mais quel est le sens exact de ce principe appliqué aux grandeurs? Montrons-le sur la première grandeur 
physique que mentionne l' Almageste, l'arc qui sépare les tropiques. Préalablement, pour des raisons d'utilité immédiate ${ }^{4}$, Ptolémée dresse une table de correspondance entre arcs croîssant de $1 / 2^{\circ}$ de 0 à $180^{\circ}$ et cordes sous-tendues correspondantes ${ }^{5}$. Or bien que ces correspondances soient démontrées géométriquement, l'auteur précise qu'il "utilise le système sexagésimal à cause de la difficulté des fractions" et qu'il "pousse les multiplications et les divisions en visant toujours à obtenir une approximation qui ne laissera pas d'erreur digne de considération en ce qui concerne la précision de nos sens"6. On ne s'étonnera donc pas qu'après avoir exposé avec soin la construction de l'instrument requis pour observer l'inclinaison de l'écliptique sur l'équateur, il donne en termes modernes la valeur de ses propres observations: "l'arc entre les points tropiques est toujours supérieur à $47^{\circ} 40^{\prime}$ mais inférieur à $47^{\circ} 45^{\prime \prime \prime}$. Ainsi les premières grandeurs physiques attestées dans l'astronomie grecque sont assorties de la mention explicite de leur caractère approché, la précision supérieure fixée n'est autre que le seuil d'acuité visual dans la discrimination des angles.

L'approximation à l'oeuvre en physique ne diffère pas seulement de son analogue dans les organes des sens par la précision quantitative qu'appelle la mesure des grandeurs. Les seuils sont innés et donc immuables aux accidents près de l'évolution qui, sous peine d'extinction, ne tolère les variations que dans la mesure où elles sont compatibles avec la survie de l'espèce. Au contraire, les limites de précision dont est effectée l'observation des grandeurs physiques sont, par définition, variables. Même l'astronome qui observe à l'oeil nu construit des instruments pour réduire autant que possible le flottement de la vision à la constation d'une coincidence entre un point ou un contour luminex et la matérialisation d'une ligne. Surtout, l'instrumentation affranchit l'homme des seuils perceptifs. On s'est interrogé sur le progrès scientifique, on ne s'interroge pas sur le progrès des méthodes d'observation ${ }^{9}$. Et, quelque confusion que produise sur certains esprits l'idée d'une confirmation et d'une infirmation approchées, si l'on conteste la signification du progrès dans la précision d'une décimale, on ne conteste pas ce progrès même. Aux deux bouts de la chaîne, telle est la nature de l'approximation qui caractérise l'observation faite ou prévue. Il reste à examiner la nature de la chaîne qui permet de passer de l'une à l'autre.

Pour qu'on puisse donc parler véritablement d'approximation pour la connaissance sensible, il faut qu'on ait réduit son objet aux rapports entre grandeurs physiques. Mais grandeurs et rapports entre grandeurs constitutent l'un des chapitres essentiels des mathématiques. Il importe donc de préciser le rôle des mathématiques en physique. Il ne suffit pas, en effet, de comparer les lois et les théories physiques aux anticipations spontanées de la perception en disant que celles-là explicitent des systèmes d'opérations semblables à celles que nous supposons lorsque nous parlons métaphoriquement des calculs du système nervuex. Il faut distinguer l'approximation propre aux grandeurs physiques de l'approximation 
mathématique. Il faut encore montrer comment la connaissance sensible, spécifiée par sa distinction d'avec la mathématique, reste néanmoins en mesure de bénéficier de son concours.

La réponse à la première de ces questions est la plus aisée. Lea mathématiques proprement dites, que Félix Klein appelait les mathématiques de la précision ${ }^{10}$, posent et résolvent sans reste le problème de l'approximation $1 \%$ en développant de façon exacte un nombre ou une fonction, $2^{\circ}$ / en estimant l'erreur commise quand on tronque une expression approchée en négligeant les termes, éventuellement en nombre infini, à partir d'un certain rang. L'approximation physique, quant à elle, est déterminée par les moyens d'observation dont le savant dispose à un moment donné. Cette détermination est entièrement contingente par rapport à l'approximation mathématique qui seule peut -généralement de façon rétrospective- éclairer sur sa nature et sur sa validité. Par exemple on démontre que, dans le cas du soleil (ou de la terre) les systèmes de Ptolémée, de Copernic et de Kepler conduisent aux mêmes valuers de l'anomalie vraie à 0 , 5 près, ụne différence que les Anciens n'auraient pas pu soumettre au critère de l'observation, et ceci parce que, lorsqu'on développe cette anomalie en fonction de l'excentricité et de l'anomalie moyenne, les deux premiers termes du développement sont identiques dans les trois systèmes et les différences qui apparaissent à partir du troisième terme sont négligeables étant donné la faible excentricité de l'orbite ${ }^{11}$. On voit ainsi la décision du physicien, qui doit choisir entre plusieurs hypothèses, être suspendue au progrès dans l'approximation d'une grandeur.

La solution de cette première question conduit à la solution de la seconde, apparemment plus difficile. On se demande maintenant si et comment la connaissance sensible, bornée aux rapports de grandeurs et relative à une approximation, peut bénéficier des apports des mathématiques pures. L'équation de Kepler, qu'on vient d'invoquer, permet de répondre. Les mathématiques, en effet, ne sont pas seulement un langage bien fait, en ce qu'elles éliminent les équivoques des langues naturelles. Elles le sont surtout en établissant des dictionnaires de traduction interne entre théories mathématiques ${ }^{12}$. Ces dictionnaires sont partout présents dès que la science atteint quelque développement. Contentons-nous de citer la correspondance par dualité entre le théorème de Pascal et le théorème de Brianchon démontrée par Poncelet du point de vue de la géométrie projective et la correspondance entre les trois géométries à courbure constante qui résulte des dictionnaires dressés par les géomètres du XIXème siècle et en particulier par Poincaré. C'est encore Ptolémée qui nous fournit le premier exemple d'un tel dictionnaire en cinématique céleste en établissant les conditions auxquels les paramètres d'un système excentrique et d'un système épicyclique doivent obéir pour être équivalents ${ }^{13}$.

La comparaison entre les systèmes du monde au moyen du développement en 
série trigonométrique de l'anomalie vraie et du rayon-vecteur, dues à l'équation de Kepler, illustre ces dictionnaires, dont on ne tardera pas à apercevoir l'importance fondamentable pour la méthode de la physique.

\section{Contraintes que fait peser l'approximation sur observation, lois et théories}

L'enquête sur ces contraintes paraît se heurter à une objection préalable : est-il légitime de distinguer observation, loi et théorie physique? On accordera aisément le caractère conventionnel de la distinction entre loi et théorie. Un principe théorique n'est qu'une loi générale; une collection de tels principes définit une théorie. Est-il plus sérieux d'objecter que toute observation incorpore une théorie et d'en conclure que le rapport entre théorie et observation est circulaire? Pour mesure les longueurs, on choisit une règle de métal de longueur unité. Cette règle ne conserve la même longueur que si sa température ne varie pas. Pour vérifier la constance de la température, on mesure si tel corps étalon s'est dilaté. Pour mesurer la dilatation, on se sert de l'unité de longueur. Les difficultés logiques que l'argument met en évidence tiennent à ce qu'on demande à la mesure une précision absolue. Elles s'évanouissent dès qu'on explicite les marges d'approximation et qu'on interprète les corrections comme des emboîtements progressifs de ces marges. Toute mesure incorpore une théorie ; mais le contrôle expérimental des paramètres théoriques en jeu dans l'observation permet d'isoler le phénomène objet d'étude en construisant éventuellement à cet effet des domaines critiques d'expérience.

Examinons donc la contrainte de l'approximation sur l'observation. Elle tient à la nécessité de s'en tenir à une définition opérationnelle des gradeurs physiques. Le sens commun réagit au progrès des mesures en postulant que chaque fois que nous mesurons une grandeur les défauts de nos appareils introduisent une erreur inconnue; nous trouvons un nombre a, que nous appelons valeur de la grandeur et tout ce que nous pouvons dire c'est qu'il existe un nombre réel $\alpha$, qui représente la mesure vraie et exacte de la grandeur, et que $\alpha$ est situé dans l'intervalle

$$
a-\varepsilon \leq \alpha \leq a+\varepsilon
$$

où $\varepsilon$ représente un nombre positif petitit. C'est ce préjugé qu'il faut déraciner. L'expérience fournit et elle ne peut fournir pour une grandeur unique qu'un ensemble fini de nombres rationnels $\left\{a_{i}\right\}$ correspondant aux différentes observations. Il arrive que plusieurs observations fournissent le même nombre, ce qui signifie seulement que le résultat de ces observations est indiscernable, compte tenu de la précision de l'instrument. De l'ensemble $\left\{a_{i}\right\}$ on tire une valeur, $a$, dite valuer vraie, en appliquant le calcul statistique des erreurs. Mais, quelque soit le degré de confiance qu'on est en droit d'attribuer à cette valeur, il est évident qu'elle reste 
affectée par la marge d'imprécision liée à l'instrument. Ce n'est jamais un nombre, mais un intervalle de nombres qui lui correspond. Puisque l'oeil nu ne peut, dans les meilleures conditions, discriminer mieux que 16" d'arc, il faudra regrader toute estimation $a$ de la grandeur d'un arc par l'oeil comme une expression abrégée pour l'expression " $a \pm 16$ ", seule valeur d'observation qui ait un sens à ce niveau.

Passons aux lois et aux théories. Il suffit de tirer les conséquences résultant des contraintes qui pèsent sur l'approximation des grandeurs pour assigner les contraintes qui pèsent sur la forme élémentaire de la loi, l'égalité. Une égalité physique entre deux grandeurs $A$ et $B$, mesurées par deux nombres, $a$ et $b$ devra se distinguer de l'égalité mathématique correspondante, $a=b$, puisque les mesures de $A$ et de $B$ sont des nombres affectés respectivement de deux marges d'imprécision $\pm \varepsilon_{1}$ et $\pm \varepsilon_{2}$. Puisqu'au point de départ les mesures a de $A$ et $b$ de $B$ correspondent aux intervalles $a^{\prime}=a \pm \varepsilon_{1}$ et $b^{\prime}=b \pm \varepsilon_{2}$, l'égalité physique aura donc pour signication réelle :

$$
\begin{gathered}
a=b \pm \varepsilon \\
\operatorname{avec}^{16} \\
\varepsilon=\left|b^{\prime}-a^{\prime}\right|+\varepsilon_{1}+\varepsilon_{2}
\end{gathered}
$$

Par conséquent, lorsque nous disons qu'une grandeur $A$ est une fonction physique $f$ d'une grandeur $B$, l'expression mathématique $f(a, b)=0$ que nous employons abrège l'expression du procédé suivant. Reportons graphiquement en abscisse les valeurs obtenues $a$ pour $A$ et en ordonnée les valeurs obtenues $b$ pour $B$. Par chacun de ces points, $(a, b)$, pris comme origines, nous construisons les segments $\pm \varepsilon_{1}$ parallèles à l'axe des abscisses et $\pm \varepsilon_{2}$ parallèles à l'axe des ordonnées, où $\varepsilon_{1}$ et $\varepsilon_{2}$ indiquent la précision des mesures respectives de $A$ et de $B$. Si les points $(a, b)$ sont assez rapprochés, les petites croix ainsi déterminées par les intervalles $\left(a^{\prime}\right.$, $\left.b^{\prime}\right)$ individualisent une bande à l'intérieur de laquelle la courbe représentative de $f\left(a^{\prime}, b^{\prime}\right)=0$ doit être contenue ${ }^{17}$.

Si par un nombre fini de points, il est toujours possible de faire passer un nombre infini de courbes, dans une bande il est toujours possible d'enfermer une infinité de courbes assujetties à respecter les contraintes d'approximation qu'on a dites. En principe il faudrait donc faire intervenir ici l'ensemble des courbes contenues dans la bande, cet ensemble étant déterminé par l'ensemble des phénomènes étudiés et des marges de précision imposées à la mesure des grandeurs ${ }^{18}$. On demanderait alors à un critère de simplicité de choisir dans cet ensemble de courbes. Hantés par les objections irréfutables faites par Hume à l'induction physique, les philosophes empiristes ont, sans la découvrir, cherché une définition générale de la simplicité. Mais chacun sait ce qui est simple dans un cas particulier, c'est-à-dire une fois fixés et l'état des connaissances et la précision de l'approximation des grandeurs, et c'est 
ce qui suffit si toutes les lois qui peuvent se présenter en physique se rangent dans l'un des quatre cas suivants :

1/ Les mesures de deux grandeurs donnant deux suites de nombres, on cherche par tâtonnement à établir entre ces nombres un rapport de proportionalité ou une relation binomiale. Les Grecs connaissent ainsi les deux premières formes de la loi de réfraction ${ }^{20}: i=n r$ et $r=a i-b i^{2}$. Il est probable qu'ils s'aperçurent que ces lois n'étaient acceptables que pour des valuers petites de l'angle d'incidence. On rétablit l'universalité de la loi en restreignant son domaine : elle est valable, dira-ton $^{21}$, non pas quel que soit l'angle d'incidence, mais lorsque cet angle varie de $0^{\circ} \grave{a}$ $30^{\circ}$.

2) Une préparation expérimentale très particulière permet de simplifier le phénomène et de fixer une relation élémentaire entre deux grandeurs qui est universelle sous la condition de cette implication. Ainsi procèdent Mariotte et Boyle. En préparant un gaz très raréfié dans un cylindre, on montre que la pression est inversement proportionnelle au volume d'un gaz "parfait". Les précautions dont il faut s'entourer quand on comprime le gaz pour ne pas élever la température rappellent à l'expérimentateur que l'universalité de sa loi dépend de conditions très spéciales et, à vrai dire, abstraites.

3/ L'assignation des premières lois physiques dépend souvent d'un principe théorique qu'imposent des considérations de simplicité métaphysique. Ainsi la simplicité du mouvement circulaire impose l'hypothèse de la trajectoire circulaire apparente du Soleil autour de la Terre. Cette hypothèse est une très bonne approximation ${ }^{22}$.

4/ En général, le physicien dispose d'une loi héritée de la tradition ou d'une pluralité de telles lois. Ces lois sont par exemple vérifiées à une approximation donnée, qui ne permet d'ailleurs pas de les départager. Un progrès dans la précision de la mesure oblige à les abandonner. Ainsi Kepler, désirant déterminer l'orbite de Mars, rencontre deux hypothèses faisant de cette orbite soit un mouvement épicyclique uniforme dont le centre est lui-même animé d'un mouvement circulaire uniforme (modèle copernicien), soit un mouvement circulaire avec équant ptolémaïque mais dont le Soleil, et non plus la Terre, occupe le foyer réel (modèle de Tycho-Brahé). La précision des mesures de Tycho-Brahé permet d'écarter les deux modèles. Le modèle de Tycho est moins éloigné des observations que celui de Copernic. Kepler essaie donc de l'amender. Il imagine une hypothèse complémentaire qui renonce à l'égalité entre l'excentricité et la distance du centre de l'orbe à l'équant. L'observation réfute l'hypothèse. Il faut revenir à la bissection de l'excentricité. Kepler tente de combiner la bissection avec les épicycles coperniciens; les orbites ainsi produites sont des ovales ${ }^{23}$. Tandis que les trajectoires réelles auxquelles Copernic aboutissait étaient des ovales, mais que Copernic ne s'intéresse qu'aux mouvements élémentaires tous circulaires uniformes, Kepler étudie directement les grandeurs accessibles à l'observation, quand on tient compte du mouvement de la Terre ${ }^{24}$; il 
choisit comme variables indépendantes le rayon-vecteur et l'anomalie vraie. Ses tâtonnements le conduisent à une formule liant ces deux grandeurs en fonction de l'excentricité et de la demi-distance aphéliepérihélie dans laquelle il ne reconnaît pas l'expression approchée d'une ellipse ${ }^{25}$. Finalement, Kepler choisit l'ellipse comme étant l'ovale le plus simple et le mieux connu après le cercle. Il l'identifie enfin avec l'approximation de la courbe à laquelle l'avaient mené ses tâtonnements. Que la simplicité joue le rôle de critère de sélection est notable pour deux raisons. La loi des mouvements elliptiques fut contestée, précisément parce qu'elle mettait fin au principe de simplicité des orbites circulaires, mais Kepler n'a pas manqué d'affirmer, avec quelque raison, que son hypothèse était en réalité plus simple que cells qui avaient précédé la sienne ${ }^{26}$. En second lieu, cette simplicité n'est définie que relativement à l'état des connaissances et à la précision des mesures. La loi de Kepler a pu supplanter ses devancières parce que, en portant à 2' la limite de l'approximation des mesures, Tycho-Brahé, en portant à 2' la limite de l'approximation des mesures, Tycho-Brahé contraignait à refuter des hypothèses qui avaient pour conséquence un écart de $8^{\prime}$ entre prédictions et observations ${ }^{27}$. On a d'ailleurs justement remarqué que "si Kepler a pu conclure que l'orbite de Mars était une ellipse, c'est parce que la précision des observations ne dépassait pas 1'. Une précision plus grande lui aurait fait reconnaître l'irrégularité de l'orbite de Mars dont la trajectoire n'est qu'approximativement une ellipse"28.

\section{Vérité et signification}

Un énoncé de physique, qu'il exprime une observation, une loi ou une théorie, n'est donc complet que lorsqu'on mentionne sa marge d'approximation. De ce principe résultent deux sortes de conséquences touchant les notions de vérité et de signification dans leur rapport à la connaissance sensible.

En mathématiques, l'égalité entre une fonction trigonométrique et son développement infini en série de puissances est une égalité absolue ${ }^{29}$. Il en va de n̂eme pour une équivalence modulo un groupe de transformation, équivalence qui se réduit d'ailleurs à une égalité entre ensembles ${ }^{30}$. Au contraire, en physique, deux grandeurs ne sont égales qu'à une marge près, $\varepsilon: a=b \pm \varepsilon$. La même relativité à une marge s'impose pour l'équivalence entre lois et théories. Ces marges changent. Lorsque, comme il arrive en histoire, ce changement est un progrès dans la précision, à la marge $\varepsilon^{\prime}<\varepsilon$ toutes les différences et les disparités établies relativement à $\varepsilon$ sont conservées. En revanche, la meilleure précision de $\varepsilon^{\prime}$ peut détruire les égalités et les équivalences relatives à $\varepsilon$.

Les conséquences de cette simple remarque sont si importantes qu'on fera bien de les illustrer en revenant sur l'exemple keplerien. La démarche keplerienne pour Mars revient à démontrer que, données les observations de Tycho-Brahé, la bande 
construite à partir d'elles contient l'excentrique ptolémaïque pour l'approximation ptolémaïque de $10^{\prime}$, ne la contient pas pour l'approximation de 2 ' propre à Tycho, mais, pour la même approximation, contient l'ellipse kepleriene correpondante. Une loi vérifiée à $\varepsilon$ près peut donc être infirmée à $\varepsilon^{\prime}$ près si $\varepsilon^{\prime}<\varepsilon$. C'est qu'à la différence d'une loi mathématique, une loi physique n'est jamais universelle. La quantification sur les valeurs des grandeurs est toujours implicitement limitée.

Quels sont donc les relations de la vérité et de la quantité ? Convenons d'appeler $M$ la propriété d'appartenir aux points de l'orbite de Mars, $P$, celle d'appartenir à l'excentrique ptolémaïque de Mars, $K$ celle d'appartenir à l'ellipse keplerienne de Mars. Convenons qu'un quantificateur universel suivi de la mention de la marge d'approximation $\varepsilon$ devra être compris relativement à cette marge. Par exemple les signes:

$$
\text { (x) } 10^{\prime}
$$

signifieront: "pour tous les $x$ discernables à 10 près". En d'autres termes, les expressions :

$$
(x) \varepsilon f x \quad \text { et }(\exists x) \varepsilon f x
$$

abrègent les expressions :

$(x)$ ( $x$ est déterminé à une marge d'approximation $\geq \varepsilon \supset f x$ )

et

$$
(\exists x) \text { ( } x \text { est déterminé à une marge d'approximation } \geq \varepsilon \cdot f x) .
$$

Les trois assertions kepleriennes s'écriront alors:

$$
\begin{aligned}
& (x) 10^{\prime}(P x \supset M x) \\
& (\exists x) 2^{\prime}(P x . \sim M x) \\
& (x) 2^{\prime}(K x \supset M x) .
\end{aligned}
$$

Lorsqu'on omet la mention essentielle de la marge d'approximation on s'expose à plusieurs sortes de paradoxes. $1^{\circ} /$ Il faudra dire que lorsqu'une loi ou une théorie physique établie à une marge $\varepsilon$ est remplacée à une marge $\varepsilon^{\prime}<\varepsilon$ par une loi ou une théorie nouvelle, elle est infirmée. $2^{\circ} /$ Deux hypothèses rivales et incompatibles à la même marge $\varepsilon$ devront d'abord être dites toutes deux vraies, puis toutes deux fausses quand elles sont supplantées par und nouvelle hypothèse à la marge $\varepsilon^{\prime}<\varepsilon$. $3^{\circ}$ / Lois et théories peuvent toujours être infirmées sans pouvoir être jamais confirmées. Une fois la vérité relative à la quantité sensible, c'est-à-dire à la précision, les paradoxes s'évanouissent. Il n'y a plus changement de valuer de vérité, ni asymétrie du vrai et du faux ${ }^{31}$.

Le célèbre problème de l'induction humienne se resserre. Nulle opération logique, dit Hume, ne permet de passer de quelques à tous. Mais les lois physiques ne sont pas des implications formelles. Ce sont des universelles bornées. Le 
problème consiste à passer de tous à quelques en abaissant la borne, puis à retrouver tous à l'intérieur de la borne nouvelle. Ce dernier pas inductif résulte d'une découverte qu'aucune règle logique n'est à même d'exprimer, mais qui doit respecter la contrainte de l'approximation. Le principe de correspondance, dû à Bohr, exprime, en ce qu'il a de valide, cette contrainte : la loi ou la théorie nouvelle doit permettre, lorsqu'on relâche la marge de précision $\varepsilon^{\prime}$ et qu'on retourne à l'ancienne contrainte $\varepsilon$, de retrouver l'ancienne loi valable à $\varepsilon$ près ${ }^{32}$.

C'est ici que le physicien tire profit des dictionnaires de traduction et plus particulièrement des développements en série que lui fournit le mathématicien. Ainsi l'équation de Kepler permet de développer anomalie vraie et rayon vecteur d'une planète en fonction de l'excentricité, de l'anomalie moyenne et de la demidistance périhélie-aphélie pour les trois systèmes du monde. En fixant la valeur de $\varepsilon$ on determine alors dans quelles limites les différents systèmes du monde coïncident, c'est-à-dire font des prévisions indiscernables, ou divergent, c'est-à-dire sont discernables par l'observation.

A vrai dire il est beaucoup plus difficile d'établir comment la mécanique quantique permet de retrouver la mécanique classique à la limite où l'on n'a pas à tenir compte du quantum d'action que de dériver les lois de Kepler à partir des principes de Newton. La correspondance réussit localement comme il arrive pour les équations d' Ehrenfest. La correspondance globale reste, pour le moment, hors de portée. Le principe de complémentarité fait assez voir intuitivement les raisons de la difficulté.

C'est donc en principe que les théories physiques sont toujours comparables au point de vue de la vérité. De plus, tant que nous ignorons jusqu'a' quelle limite une théorie est valide, toute autre théorie, s'il en est, qui possède le même pouvoir prédictif en est en fait indiscernable. Si deux théories sont réellement distinctes, il existera cependant une prédiction de l'une qui n'est pas celle de l'autre. La décision attendra aussi longtemps que l'art de la mesure n'aura pas atteint la décision requise.

Mais ce n'est pas la vérité qui pose des problèmes difficiles à la comparaison. Ce n'est pas elle, parce que la vérité est du côté de l'extension et que la physique est extensionnelle. Les difficultés commencent quand on s'interroge sur la signification des propositions de la physique et, en fin de compte, sur la nature des concepts qui entrent dans la comparaison de deux lois ou de deux théories. Rien n'assure en effet que si une loi ou une théorie vérifiée à $\varepsilon^{\prime}$ près confirme la vérité d'une loi ou d'une théorie différente vérifiée à $\varepsilon>\varepsilon^{\prime}$, cette confirmation entraîne, pour les concepts correspondants, quelque convergence de compréhension ${ }^{33}$. La comparaison des systèmes astronomiques antiques pourrait induire en erreur, en fixant l'attention sur deux variables indépendantes qu'on retrouve dans tous les systèmes et qui sembles assurer l'univocité de la comparaison. Mais comparons Kepler non plus à Ptolémée ou à Copernic, mais à Newton. La troisième loi de Kepler établit un rapport de 
proportionalité entre carrés des temps et cubes des distances. Newton fait intervenir les masses qui ne figurent pas dans les équations de Kepler. De l'équation de Newton :

$$
\frac{A^{3}}{T^{2}}=\frac{\varepsilon(M+m)}{4 \pi^{2}}=\frac{\varepsilon M}{4 \pi^{2}}\left(1+\frac{m}{M}\right)
$$

on passe à celle de Kepler :

$$
\frac{A^{3}}{T^{2}}=K
$$

en posant $\frac{\varepsilon M}{4 \pi^{2}}=K$ et en négligeant la qualité $\frac{m}{M}$. Dans le cas de la Terre et du Soleil, cette dernière quantité est égale à $\frac{1}{300,000}$. Il y a bien comparabilité de la vérité. Mais on ne voit pas comment le concept de gravitation qui est la raison d'être dynamique de la loi cinématique se transformerait en concept de constante quand on borne la marge d'approximation. Kepler douait bien les corps du système solaire d'une moles, d'un volume matériel. Ce volume matériel nest pas und masse. Kepler lui attribuait une force magnétique qui ne peut être assimilée à la gravitation ${ }^{34}$.

Que reste-t-il donc du concept newtonien lorsqu'on passe à l'approximation keplerienne? Un rapport entre masses étant négligé et négligeable, il reste un rapport entre termes représentant des constantes. Ce rapport, d'où le concept de masse disparaît, devient une constante du système solaire. De même lorsqu'en connaisance de cause on assimile l'ellipse keplerienne à un excentrique, on néglige la différence entre grand et petit axe. Dans les deux cas, on réduit volontairement une information pour des raisons de simplicité et d'économie de pensée. Dans les deux cas, l'astronome connaît la limite de validité de l'approximation. Il n'utilise la loi la plus simple que parce qu'il sait que les différences supplémentaires qu'il obtiendrait à partir de la loi plus compliquée seraient sans objet pour l'observation.

Un concept a une extension et une compréhension (ou une signification). A une extension unique correspondent des compréhensions multiples, à une compréhension unique une extension unique. Prenons garde cependant qu'en physique tous les concepts reçus sont des concepts mathématiques assortis de conditions d'approximation. Ce sont par exemple des concepts de grandeurs et des concepts de fonctions. Or les mathématiques sont un langage extensionnel, où l'on peut substituer salva veritate une expression à und autre expression à condition qu'elles aient même extension, même si leurs significations sont différentes. De ce fait, les concepts mathématiques, malgré leur différence de sens, sont substituables s'ils ont même extension. Nous pouvons réduire une loi complexe à la loi simple approchée correspondante, précisément parce que l'extension de la première est partie propre de 
l'extension de la seconde.

On a vu Kepler ne reconnaissant pas le concept approché d'ellipse dans l'équation sur laquelle il était, pour ainsi dire, tombé dans ses calculs sur Mars. La reconnaissance d'un concept est encore différente de sa compréhension. Elle est délibérément subjective. Mais qu'on songe aux difficultés que recèle la lecture d'une équation. N'est-il pas remarquable que c'est Born, non Schrödinger qui a su interpréter l'équation de ce dernier? Et quoique la compréhension d'un concept paraisse se distinguer de la reconnaissance de cette compréhension comme quelque chose d'objectif se distingue de quelque chose de subjectif, nul logicien n'a pu donner une définition satisfaisante de la distinction. La compréhension des concepts est assurément nécessaire au mathématicien pour penser ses objets. Mais, ces objects étant en principe réductibles à des relations entre ensembles, on imagine un esprit divin capable d'apercevoir ces relations extensionnelles sans recourir au secours des concepts.

Le physicien, de même, a besoin de concepts pour décrire la nature. Mais la nature elle-même ne se soucie pas de nos moyens de description. L'ordre qu'elle révèle a trait aux extensions de ces concepts. Ces extensions sont comparables. Ce qui s'oppose à la comparaison, ce sont les compréhensions, et les compréhensions ne sont probablement pas entièrement séparables de la subjectivitét ${ }^{35}$.

\section{Conclusion}

Distinguons en physique deux parts : les observations, les lois et les théories dont nous connaissons à la fois les limites de confirmation et d'infirmation, les observations, les lois et les théories dont nous ignorons les limites d'infirmation. La nature des problèmes que nous avons à résoudre nous impose le choix de la part à laquelle nous avons recours. Mais lorsqu'il a recours à la première, c'est le cas quand il lance un satellite, le physicien fonde ses précisions sur un appareil théorique qui, parce que ses limites sont assignées, est utilisé pour son utilité sans conserver le pouvoir de synthèse explicative dont il jouissait tant que l'on ignorait ces limites. Les lois de Newton sont réduites à des procédés de calcul ; elles n'expliquent plus l'univers. En d'autres termes, si les relations extensionnelles auxquelles ces lois se réfèrent conservent leur plein emploi, on laisse en friche la compréhension des concepts que ces lois expriment. L'histoire est au principe de cette décantation. Elle donne son congé à la physique théorique. Elle ne retient que la physique mathématique. Celle-ci est la marque de la nature (puisque c'est la nature qui fixe les ordres de grandeur et qui délimite la validité des approximations). Celle-là est l'empreinte de l'homme. Il est juste que demeure ce que la nature détermine et que s'efface la marque de l'homme.

En quoi consiste, précisément, la spécificité d'une théorie? Il est difficile de le 
dire. Newton regrettait de n'avoir pu, pour la science, forger d'hypothèses adéquates. Par hypothèses, il entendait qualqu' explication mécaniste, du type de celle des tourbillons, quelque modèle parlant aux yeux et proposant une image sensible plausible de la causalité physique. On voit, par conséquent, qu'une théorie peut subsister quand fait défaut tout schème sensible. Cependant, nous pouvons encore penser la force d'attraction à distance que nous n'imaginons pas. Donnés deux corps, leurs masses, leurs positions, leurs vitesses présentes, la théorie newtonienne détermine leur histoire passée et future. Donnés plus de deux corps, la théorie des perturbations permet de mimer le déterminisme autant qu'il est possible. Le surplus qu'apporte la théorie sur les mathématiques, la compréhension sur l'extension, ce n'est donc pas celui de l'image, mais bien plutôt celui du principe sur le concept.

Lorsqu'en 1822 Fourier publie sa Théorie analytique de la chaleur, alors qu' aucun physicien n'objecte plus à l'action à distance, il présente une physique mathématique purgée de tout ingrédient théorique. L'équation de diffusion de la chaleur ne dépend, en effet, d'aucun principe. Elle n'engage à aucun choix entre le calorique ou chaleur-substance et la théorie cinétique ou chaleur-mouvevent. Elle se résout dans l'analyse mathématique qui, à partir de coeffcients tirés de l'expérience, établit la proportionalité entre la dérivée premiére de la température par rapport au temps et sa dérivée seconde par rapport à l'espace. On connaît l'importance de ce livre, si riche pour la physique mathématique, et exclusif de toute théorie, pour le positivisme d'Auguste Comte.

Pourquoi donc ne pas être positiviste? Etre positiviste, c'est adopter pour les observations, les lois et les théories dont nous ignorons les limites d'infirmation, l'attitude spontanée du physicien à l'égard des observations, des lois et des théories dont il connaît les limites de confirmation et d'infirmation. C'est donc simplement, précéder le jugement de l'histoire. C'est obéīr à la nature, puisque, finalement, la nature effacera, dans la science, la trace de l'homme.

Cette vue austère, objective et pour ainsi dire rétrospective de la science, sans être impossible, est paradoxale. Nous ignorons les limites d'imfirmation de notre science. Cette ignorance est un fait. Nous ne savons pas borner l'universalité des lois que nous énonçons. Nous sommes certes avertis qu'une borne existe peut-être, qu'il est plausible qu'elle existe (nous ne saurions parler ici de probabilité). Mais il n'y a rien de contradictoire à penser que la théorie dans laquelle nous vivons présentement sera confrmée aussi loin que nous poussions l'approximation. Cette possiblité -si faible qu'elle nous paraisse- ajoutée à la difficulté que nous éprouvons à penser sans principe, sinon sans image, nous porte à chercher dans la compréhension des concepts un contenu auquel nous croyons au delà de la considération des extensions. Pour croire, il nous faut un minimum d'intelligibilité, sinon d'imagination. Ceci explique que ceux-là même des physiciens qui adoptent le même système 
d'équations fondamentales se disputent sur leur interprétation. L'ignorance de la limite exacte de l'extension accroît d'autant l'indétermination de la compréhension et son attrait, pour ainsi dire, irrésistible sur l'esprit.

\section{NOTES}

1. D. Krantz, R. Duncan Luce, P. Suppes, A. Tversky, Foundations of Measurement, Academic Press, New York, London, 1971, t. I, chap. 3, pp. 71-135.

2. H. Bergson, Essai sur les données immédiates de la conscience, Paris, Alcan, 1889.

3. Almageste, 1, 1. Dans ce texte, la physique (au sens d'Aristote) est une doctrine de la qualité matérielle. Nous la rejetons de ce que nous considérons précisément comme constituant la physique. Ptolémée range donc l'astronomic dans les mathématiques.

4. Almageste, I, 10.

5. Les arcs croîssant régulièrement de $1 / 2^{\circ}$, les cordes croîssent irrégulièrement par quantités qui diminuent progressivement. Dans la Table (I, 11), la première colonne indique les valuers successives de l'arc, la seconde les valeurs successives de la corde mesurée en nombre de parties du diamètre divisé en 120 parties, la troisième la trentième partie de l'augmentation de la corde assignée par rapport à la corde précédente (I, 10).

6. I, 10 .

7. I, 12. Ptolémée ajoute: "Et de ceci il résulte à peu près le même rapport que celui d'Erathosthène et que celui qu'utilisait Hipparque. En effet l'arc entre les tropiques se trouve être très proche de 11 des 83 parties du méridien". On vérifie que $\frac{360}{47} \simeq \frac{83}{11}$, puisque $360 \times 11=3960,83 \times 47=3961$. On voit l'attention que Ptolémée porte aux observations de ses prédécesseurs.

8. Estimée à 16 secones d'arc la nuit et dans le bleu par J.-Cl. Pecker (Clefs pour l'Astronomie, Seghers, Paris, 1981, p. 44), la limite pratique de précision de l'astronomie à l'époque de Ptolémée est estimée à.

9. "Avec un objectif de $6 \mathrm{~cm}$, l'oeil sépare...2" d'arc. Au bout d'un télescope de $60 \mathrm{~cm}$ l'astronome sépare donc environ $0,2^{\prime \prime}$, en principe. Avec le téléscope de $6 \mathrm{~m}$, si tout était conforme au calcul. .., il séparerait 0, 02"” (Pecker, 1981, p. 48).

10. F. Klein, Elementarmathematik vom höheren Standpunkte aus, 3 vol., vierte Aufl, Berlin, Springer, 1968; J. Vuillemin, "Vérité partielle ou approximation de la vérité, La Gazette des Sciences Mathématiques du Québec, vol. VII, $\mathrm{n}^{\circ}$ 2, janvier 1983, (pp. 730), pp. 9-13; La nature de la vérité scientique, Archives de l'Institut international des sciences théoriques, CIACO, Louvain-La-Neuve, 1985, pp. 123-139.

11. L'excentricité $=0,016$. Les trois systèmes donnent respectivement

$$
\begin{aligned}
& v=M+2 e \sin M+2 e^{2} \sin 2 M+\ldots \\
& v=M+2 e \sin M+e^{2} \sin 2 M+\ldots \\
& v=M+2 e \sin M+\frac{5}{4} e^{2} \sin 2 M+\ldots
\end{aligned}
$$

( $v=$ anomalie vraie, $\boldsymbol{e}=$ excentricité, $M=$ anomalie moyenne) (A. Danjon, Astronomie Générale, Blanchard, Paris, 1986 (nouvelle édition), p. 177).

12. J. Vuillemin, "Internal translations and reality in science", Sprache und Ontologie, Akten des 6. Internationalen Wittgenstein Symposiums, 23. bis 30. August 1981, Kirchberg/Wechsel (Osterreich), Wien 1982, Hölder-Pichler-Tempsky, pp. 313-326.

13. Ptolémée, Almageste, III, 3 . Pour des exemples d'application, Almageste, IV, 5 ; XII, 
1.

14. J. Vuillemin, "Physique quantique et philosophie", Le monde quantique, éd. S. Deligeorges, Paris, Le Seuil, 1984, p. 210.

15. G. Toraldo di Francia, L'imagine del mundo fisico, Torino, Einaudi, 1976, p. 45.

16. Toraldo di Francia, 1976, p. 49.

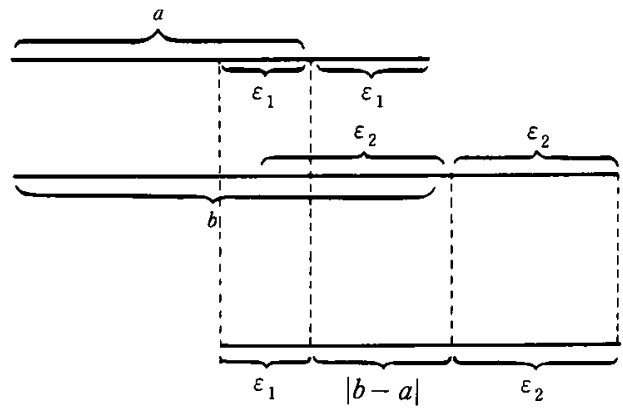

17. Je suis à la lettre Toraldo di Francia, 1976, pp. 55-56.

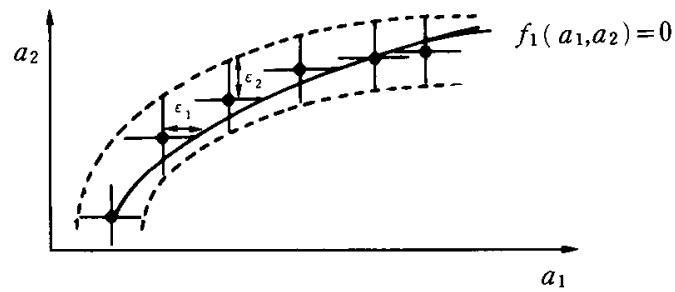

C'est cette bande que F. Klein appelait Bandkurve.

18. Ainsi procède Toraldo di Francia, 1976, pp. 56-57.

19. Le critère général de Popper ( La logique de la découverte scientifique, trad. ThyssenRutten et Devaux, Paris, Payot, 1973, pp. 136-145), le degré d'infirmabilité, suppose bien défini le degré d'infirmabilité.

20. Où $\mathrm{n}, a, b$ sont des constantes. La première loi est adoptée par des opticiens postérieurs à Ptolémée. La seconde est due à Héron (et a été attribuée à Ptolémée) (J. Vuillemin, 1983, note 24, pp. 23-27).

21. Pour $30^{\circ}$ l'écart de la loi $\mathrm{i}=\mathrm{nr}$ avec la loi de Descartes est égal à $1 / 2^{\circ}$.

22. Voir note 11.

23. Voir les articles de P. Russo et de J.-Cl. Pecker, in Quatrième Centenaire de la naissance de Johannes Kepler, Société Astronomique de France, Paris, 1973, pp. 92-94 et pp. 109-117.

24. Donc en rapportant ces grandeurs au Soleil comme origine. Préalablement, Kepler réduit l'excentricité de l'orbite terrestre de 0,035 (Ptolémée) à 0,018 .

25. (1) $\quad r=a(1-e \cos v)$

(B. Morando, in Quatrième Centenaire..., 1973, p. 0).

L'expression exacte de l'ellipse est:

$$
r=a(1-e \cos E)
$$


où $E$ est l'anomalie excentrique. A partir des équations respectives de l'ellipse et du cercle exinscrit, on montre que :

$$
\cos v=\frac{\cos E-e}{1-e \cos E} .
$$

En substituant l'expression de droite de (2) dans (1), il vient, en négligeant les termes en $e^{2}$ et $e^{3}$ au fur et à mesure qu'ils apparaissent:

$$
\begin{aligned}
r & =a\left(1-e \frac{\cos E-e}{1-e \cos E}\right)=a\left[1-e \frac{(1+e \cos E)(\cos E-e)}{1-e^{2} \cos ^{2} E}\right] \\
& \simeq a[1-e(1+E \cos E)(\cos E-e)]=a\left(1-e \cos E+e^{2}-e^{2} \cos ^{2} E+e^{3} \cos E\right) \\
& \simeq a(1-e \cos E) .
\end{aligned}
$$

26. Lettre à Fabricius du ler août 1607 (cité par P. Russo, 1973, p. 84). Kepler écrit: "La simplicité de la nature ne doit pas être mesurée par notre imagination".

27. "Pour moi, dit Kepler, à quí la grâce divine a donné dans Tycho-Brahé un observateur d'une valeur telle que ses observations nous révèlent l'erreur de 8' commise par Ptolémée, il (me convient) d'accepter avec reconnaissance ce bienfait de Dieu et d'en tirer profit" (cité par J.-Cl. Pecker, 1981, p. 115).

28. P. Russo, 1973, p. 82 .

29. Exemple: $\sin x=x-\frac{x^{3}}{3 !}+\frac{x^{5}}{5 !}-\ldots$

30. L'exemple paradigmatique est celui du nombre. S'il existe une correspondance biunivoque entre deux ensembles, $\mathbf{A}$ et $\mathbf{B}$, la puissance de $\mathbf{A}$ est égale à la puissance de B.

31. Naturellement, s'il s'avérait que

$$
(\exists x) 10^{\prime}(P x-M x)
$$

la loi ptolémaĩque, infirmée à 10', n'en serait pas une.

Elle serrait simplement fausse !

32. "Les essais faits, ̀̀ l'intérieur de la théorie des quanta, pour appliquer chaque concept classique en l'interprétant en sorte de satisfaire à l'exigence de lier immédiatement la description quantique au type de description ordinaire dans le domaine limite dans lequel nous pouvons faire abstraction du quantum d'action, trouvèrent une expression dans ce qu'on appelle le principe de correspondance" (cité par E. Scheibe, "Zum Theorienvergleich in der Physik", Physik, Philosophie und Politik, hsggben von Meyer-Abich K.M., Hanser, München, 1982, p. 295.

33. E. Scheibe remarque que Bohr fait suivre le texte qu'on vient de citer note 32 , par les lignes suivantes: "L'applicabilité des lois électromagnétiques et mécaniques dans ce domaine limite ne peut être regardée que comme une dissimulation de la différence de principe entre les lois, qui commandent le mécanisme effectif des processus et des lois de la conception classique" (Ibid., p. 296).

34. I. Newton, Mathematical Principles of Natural Philosophy, Book 3, Prop. 6, Theorem 6, Cor. V.

35. En ce sens, Toraldo di Francia, 1976, p. 62, note 1 . 